\title{
LI. The effect of change of temperature on spontaneous ionization
}

\section{Norman Campbell}

To cite this article: Norman Campbell (1907) LI. The effect of change of temperature on spontaneous ionization, Philosophical Magazine Series 6, 13:77, 614-622, DOI:

10.1080/14786440709463637

To link to this article: http://dx.doi.org/10.1080/14786440709463637

册 Published online: 16 Apr 2009.

Submit your article to this journal

Џ Article views: 2

Q View related articles $\square$ 


\section{$\left[\begin{array}{ll}614 & ]\end{array}\right.$}

LI. The Effect of Change of T'emperature on Spontaneous Ionization. By Normar CaMpBeld, Fellow of Trinity College, Cambridge*

1. TN a recent paper $\dagger \mathrm{I}$ described some curious changes in the spontaneous ionization of a vessel which is being either heated or cooled. Since the changes appeared to have no connexion with the problem of the radioactivity of ordinary materials, which was at that time under consideration, further investigation of them was postponed. At intervals during the last two and a half years a large number of additional observations have been made: the effects can now be described with some completeness, and a tentative explanation of them offered. The changes observed are of great complexity and seem to be of little intrinsic interest, but, since other workers may be troubled by them, it is desirable to record the following notes.

\section{Experimental Arrangements.}

2. The dimensions of the tin vessel used in most of the experiments is shown in fig. 1 . The walls are maintained at a potential (about 500 volts) high enough to send a saturation current through the gas to the electrode which is connected to a Wilson tilted electroscope and initially earthed. The vessel stands on blocks of slate and is usually heated by a small gas flame placed underneath it. Since it is of the utmost importance that the insulation through which the electrode passes should be kept cool, the tube carrying that insulation is surrounded by a water-jacket through which a slow stream of water passes. This device was found to simplify considerably the effects observed.

3. The investigation has been hampered by the difficulty of obtaining consistent results. Indeed the attempt to obtain quantitative consistency has been abandoned and a general constancy in the nature of the changes observed accepted as the most satisfactory condition possible. The almost invariable sequence of changes when the vessel is first heated and then cooled may be summarized as follows.

\section{Nature of the Changes.}

4. When the source of heat is applied to the vessel the leak through the latter increases temporarily but falls to its former value in a period of the order of a minute: this period is

* Communicated by the Author.

$\dagger$ Phil. Mag. April 1905, p. 545. 
much shorter than that required for the temperature of the vessel to reach a steady state. The normal value of the leak is maintained as long as the heating is continued. When the source of heat is removed a temporary decrease of the

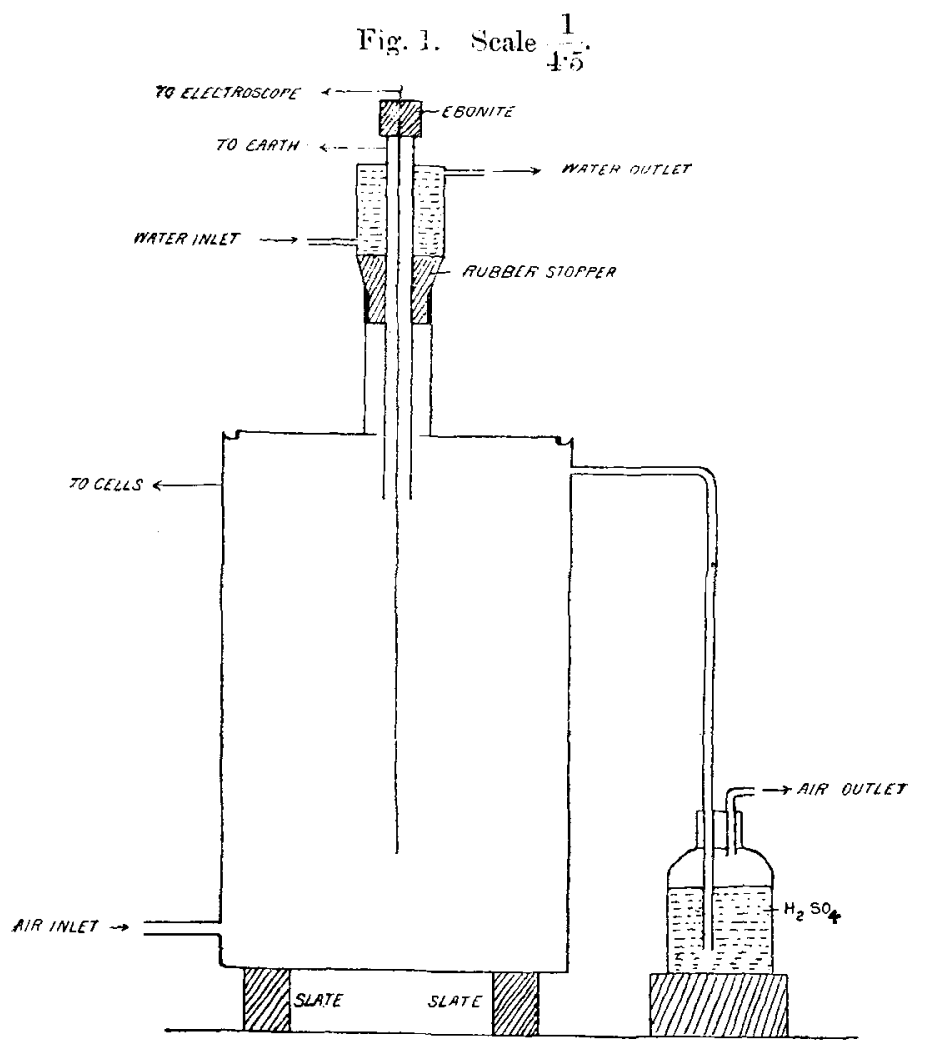

leak ensues lasting for a period of the same order as that of the increase, but, I think, a little longer. The variations of the magnitude of the changes in different experiments makes the estimation of this period very difficuit, but it may be roughly indicated as 15 seconds for the increase and 30 seconds for the decrease. After the initial decrease the leak returns to its normal value and remains constant, though the temperature of the vessel may continue to fall perceptibly for half an hour.

(The statement that the leak through the vessel when hot is equal to that through the vessel when cold is not strictly 
accurate : the "hot" leak is about 5 per cent. smaller than the "cold" leak. Since the pressure of the gas in the vessel is kept constant, the difference may be attributed to diminution of its density when heated.)

5. Such measurements as are possible indicate that the effect of heating is to canse a definite quantity of electricity of the same sign as the charge on the walls to cross from the walls to the electrode: and that the effect of cooling is to cause an equal quantity of electricity to pass in the opposite direction. The evidence for this statement will now be given.

6. The leak was measured by observing the time $t$ required for the insulated electrode to reach a known potential V : $t$ is about three minutes and $\mathrm{V}$ about $\cdot 26$ volt. When the ressel was cold individual readings varied about 8 per cent., and the mean of a long series was constant to 2 per cent.: when the vessel was hot these variations were doubled. When the effect of heating is to be observed, the electrode is insulated as usual and the flame immediately placed below the vessel: the time $t^{\prime}$ required for the electrode to reach the potential $\mathrm{V}$ is less than $t$. Similarly during the first cooling the time $t^{\prime \prime}$ is greater than $t$. Let $V$ be the capacity* of the electrode system, $Q$ the extra quantity of electricity which passes during the initial heating, and $Q^{\prime}$ that which passes during the initial cooling. Then if $t_{1}$ is the time required for the leak when the vessel is hot, $q$ the steady current when the vessel is cold, $q_{1}$ when the vessel is hot,

$$
\begin{aligned}
& \mathrm{CV}=q t=q t^{\prime}+\mathrm{Q} \text { and } \mathrm{CV}=q_{1} t_{1}=q_{1} t^{\prime \prime}+\mathrm{Q}^{\prime} \\
& \therefore \quad \mathrm{Q}=\mathrm{V}^{t-t^{\prime}}+\text { (1) and } \frac{\mathrm{Q}^{\prime}}{\mathrm{C}}=\mathrm{V} t_{1}-t^{\prime \prime} \\
& t_{1}
\end{aligned}
$$

7. The following table gives the values of $Q$ and $Q^{\prime}$ for several series of experiments performed on the same vessel at different times. The series have been specially selected, for the inconsistency between different observations is such that it is useless to draw quantitative conclusions except from those experiments which were thought from the general behaviour of the apparatus to be especially reliable. It will be seen that $Q=-Q^{\prime}$ approximately and is independent of $\mathrm{V}$ : accordingly the statement in the last paragraph is substantiated.

* Direct observations showed that the alteration of the capacity with the heating was not appreciable. 
Table I.

\begin{tabular}{|c|c|c|c|c|c|c|}
\hline V. & t. & $t^{\prime}$ & $Q / C \times 10^{3}$. & $t_{1}$. & $t^{\prime \prime}$. & $-Q^{\prime} / C \times 10^{3}$ \\
\hline$\cdot 261$ & 203 & 157 & $59)$ & 209 & 243 & 47 \\
\hline$\cdot 261$ & 207 & 155 & 65 & 210 & 259 & 59 \\
\hline$\cdot 261$ & 199 & 145 & $58>60$ & 202 & 257 & $71 \succ 60$ \\
\hline .261 & 175 & 129 & $69\}^{\circ}$ & 176 & 213 & $55\}^{\infty}$ \\
\hline .261 & 171 & 140 & 47 ) & 181 & 280 & $71)$ \\
\hline$\cdot 572$ & 471 & 403 & $83)$ & 492 & $5(1$ & 80 \\
\hline .572 & 472 & 438 & 41 & 492 & $55^{\circ}$ & 70 \\
\hline 572 & 469 & 420 & (37 $>57$ & 483 & 535 & $62 \succ 65$ \\
\hline 572 & 520 & 479 & 45 & 540 & 601 & (i5) \\
\hline $57^{2}$ & 513 & 467 & 51 & 5032 & 583 & 54 \\
\hline
\end{tabular}

8. The equality of $\mathrm{Q}$ and $-\mathrm{Q}^{\prime}$ may be tested more directly. The electrode is insulated, the flame applied for a minute and then removed: after the lapse of another minute all the heating changes are over. If $Q=-Q^{\prime}$ the total leak will be the same as if no heating had taken place. However, such observations showed that $Q^{\prime}$ was a little greater numerically than $\mathrm{Q}$. The difference between the two results may arise from the fact, that in the second of the two methods the initial cooling does not take place from a condition of thermal equilibrium.

\section{Conditions which determine the changes.}

9. The remainder of the observations were directed to determining the factors which influence the magnitude of $Q$, which may be taken as a measure of the intensity of the changes.

$\mathrm{Q}$ is independent of the direction of the current through the vessel and of the magnitude of the P.D. between the walls and the electrodes, so long as this is sufficient to send a saturation current through the gas. (The degree of saturation was such that an increase of the P.D. from 500 volts, the value usually employed, to 1000 volts produced an increase of 5 per cent. in the leak.) If the P.D is considerably less than that required for saturation the magnitude of the effect diminishos, until, when the vessel is heated with the walls and the electrode at the same potential, no charge is communicated to the latter.

10. $Q$ does not vary greatly with the material of the walls of the vessel, except in so far as these influence the ionization in the vessel (see $\$ 12$ ): experiments were made with zinc, tin, copper, and lead walls. It is also nearly 
accurate : the "bot" leak is about 5 per cent. smaller tham the "cold" leak. Since the pressure of the gas in the vessel is kept constant, the difference may be attributed to diminution of its density when heated.)

5. Such measurements as are possible indicate that the effect of heating is to cause a definite quantity of electricity of the same sign as the charge on the walls to cross from the walls to the electrode: and that the effect of cooling is to cause an equal quantity of electricity to pass in the opposite direction. The evidence for this statement will now be given.

6. The leak was measured by observing the time $t$ required for the insulated electrode to reach a known potential $V$ : $t$ is about three minutes and $V$ about $\cdot 26$ volt. When the vessel was cold individual readings raried about 8 per cent., and the mean of a long series was constant to 2 per cent.: when the vessel was hot these variations were doubled. When the eflect of heating is to be observed, the electrode is insulated as usual and the flame immediately placed below the vessel: the time $t^{\prime}$ required for the electrode to reach the potential $\mathrm{V}$ is less than $t$. Similarly during the first cooling the time $t^{\prime \prime}$ is greater than $t$. Let $V$ be the capacity * of the electrode system, $Q$ the extra quantity of electricity which passes during the initial heating, and $Q^{\prime}$ that which passes during the initial cooling. Then if $t_{1}$ is the time required for the leak when the vessel is hot, $q$ the steady current when the vessel is cold, $q_{1}$ when the vessel is hot,

$$
\begin{aligned}
& \mathrm{CV}=q t=q t^{\prime}+\mathrm{Q} \quad \text { and } \quad \mathrm{CV}=q_{1} t_{1}=q_{1} t^{\prime \prime}+\mathrm{Q}^{\prime} \\
& \therefore \quad \mathrm{Q}=\mathrm{V}^{t-t^{\prime}}{ }_{t} \quad(1) \quad \text { and } \quad \mathrm{Q}^{\prime}=\mathrm{V}_{1}-t^{\prime \prime} \quad(2) .
\end{aligned}
$$

7. The following table gives the values of $Q$ and $Q^{\prime}$ for several series of experiments performed on the same vessel at different times. The series have been specially selected, for the inconsistency between different observations is such that it is useless to draw quantitative conclusions except from those experiments which were thought from the general behaviour of the apparatus to be especially reliable. It will be seen that $Q=-Q^{\prime}$ approximately and is independent of $V:$ accordingly the statement in the last paragraph is substantiated.

* Direct observations showed that the alteration of the capacity with the heating was not appreciable. 
TABIL I.

\begin{tabular}{|c|c|c|c|c|c|c|}
\hline V. & $t$. & $t^{\prime}$. & $\mathrm{Q} / \mathbf{C} \times 10^{3}$ & $t_{1}$. & $t^{\prime \prime}$. & $-Q^{\prime} / C \times 10^{3}$ \\
\hline$\cdot 261$ & 203 & 157 & 59 & 209 & 243 & 47 \\
\hline$\cdot 261$ & 207 & 155 & $6 \tilde{5}$ & 210 & 259 & 59 \\
\hline .261 & 199 & 145 & $58\} 60$ & 202 & 257 & $71<60$ \\
\hline$\cdot 361$ & 175 & 129 & 69 & 176 & 213 & 55 \\
\hline$\cdot 261$ & 171 & 140 & $47)$ & 181 & 240 & 71 \\
\hline 572 & 471 & 403 & $83\}$ & 492 & 561 & 80 \\
\hline .572 & 472 & 438 & 41 & 492 & 552 & 70 \\
\hline 572 & 469 & 420 & $67>57$ & 483 & 535 & 62 \\
\hline .572 & 520 & 479 & 45 & 540 & 601. & (6) \\
\hline 572 & 513 & 467 & $51)$ & 532 & 583 & $54)$ \\
\hline
\end{tabular}

8. The equality of $Q$ and $-Q^{\prime}$ may be tested more directly. The electrode is insulated, the flame applied for a minute and then removed: after the lapse of another minute all the heating changes are over. If $Q=-Q^{\prime}$ the total leak will be the same as if no heating had taken place. However, such observations showed that $Q^{\prime}$ was a little greater numerically than $Q$. The difference between the two results may arise from the fact, that in the second of the two methods the initial cooling does not take place from a condition of thermal equilibrium.

\section{Conditions which determine the changes.}

A. The remainder of the observations were directed to determining the factors which influence the magnitude of $Q$, which may be taken as a measure of the intensity of the changes.

$\mathrm{Q}$ is independent of the direction of the current through the vessel and of the magnitude of the P.D. between the walls and the electrodes, so long as this is sufficient to send a saturation current through the gas. (The degree of saturation was such that an increase of the P.D. from 500 volts, the value usually employed, to 1000 volts produced an increase of 5 per cent. in the leak.) If the P.D is considerably less than that required for saturation the magnitude of the effect diminishes, until, when the vessel is heated with the walls and the electrode at the same potential, no charge is communicated to the latter.

10. Q does not vary greatly with the material of the walls of the vessel, except in so far as these influence the ionization in the ressel (see $\$ 12$ ): experiments were made with zine, tin, copper, and lead walls. It is also nearly 
independent of the gas in the vessel : air, hydrogen, carbon dioxide, and coal-gas were employed.

11. Several methods of heating the vessel were trieda small luminous gas-jet burnt from a fine glass tube, a Bunsen burner, a steam-jet, a metal box through which steam was passed, an oil-bath and a coil of wire wrapped round the vessel and carrying a current. The magnitude of the changes do not depend directly on the temperature of the source of heat, for all the above sources conld be made to give similar effects : it seems rather to be determined by the rate at which heat is commmicated to the ressel. No actual measurements of this quantity were taken, since ther would have required great experimental elaboration and did not seem likely, in the light of the only explanations of the matter that could be devised, to lead to any valuable results. In any given vessel the magnitude of the changes could not be increased beyond a certain limit whatever the source of heat employed. But by employing less powerful sources the changes might be diminished without limit. Thus in the case of the vessel described in $\$ 2$, the value of $Q$ was approximately the same whether a luminous jet $2 \mathrm{cms}$. long or' a large Bunsen burner was used; but if a jet only $1 \mathrm{~cm}$. was used, $Q$ was too small to be measured with any certainty. With the big vessel used in the experiments described in the former paper, no source of heat less powerful than a Bumsen burner produced the maximum effect.

If the vessel is turned upside down and heated on the top, the changes are very much smaller. Indeed, it was only by playing a large blowpipe-flame on the top of the vessel, at, the risk of melting the joints, that changes were obtained that could be detected with certainty.

It should be remarked that, since all methods of heating produce the changes, they cannot be due to the increase of the ionization in the air ontside the vessel owing to the presence of the flame gases.

The effect of cooling the top of the vessel by placing on it ice or liquid air was also tried. When the cooling agent was applied a temporary increase in the leak was noted, and when it was removed a temporary decrease. Hence it seems that the sign of the change is not determined by the direction of the change of temperature, but that an increase in the leak is the accompaniment of a disturbance of the normal thermal equilibrium, and a decrease of a return to that condition.

12. Q, increases with the ionization in the ressel, but not so rapidly as the ionization. When the latter was increased twenty-fold by placing uranium oxide in the ressel, $Q$ was 
raised to about twice its normal ralue. Similar effects were noticed when the air was ionized by Röntgen rars or by $\beta$-rays from radium placed outside the vessel.

13. The magnitude of $Q$ increases, both absolutely and relatively to the normal leak $q$, with an increase in the volume of the vessel and with an increase of the ratio of the heated surface to the whole surface of the vessel. In the very large flat ressel (a cylinder of 1 metre diameter and $19 \mathrm{cms}$. height) used in the former experiments, $Q^{\prime}$ may be so large that the direction of the apparent current during the initial cooling is reversed and there is a leak against the electric field (which amounted to some 50 volis per $\mathrm{cm}$.). 'The reversal of the leak was so surprising that every effort was made to discover its cause in some peculiarity of the experimental arrangements. However, it has been observed in two different vessels with no material (except the air inside them) common to both, in which the leak was measured by different methods.

14. $Q$ is increased by the presence of dust in the air; but I have never been able to reduce its value below a certain limit by blowing in air through a plug of cotton-wool two feet long. Moreover, if dusty air is used, the effects obtained on heating up the vessel a second time will be less intense than those observed at the first heating; but if dust-free air is used, alternate beating and cooling for days will not stop the change. No difference could be observed between air saturated with moisture and air dried by a long tube of calcium chloride, except that the former spoilt the insulation.

15. A few experiments were made in which the air was lept at constant volume instead of at constant pressure. The changes observed were of the same nature as before.

16. The abové summary by no means includes all the observations that have been taken, but includes all that I have been able to reduce to any sort of order. On several occasions a wholly new set of heat-changes has appeared; but when an attempt was made to investigate them later, they could not be reproduced.

\section{Explanation of the Changes.}

17. Only one explanation can be suggested which seems at all competent to account for the effects which have just been described. It may be supposed that the air of the vessel contains charged particles much heavier than the ordinary ions, possessing, in fact, a mass so large that the gravitational forces acting on them are larger than the electrical forces due to the field in the vessel. When the vessel is cold 
these particles collect on the walls, or on the floor. If a source of heat is applied, convection currents are set up which carry the particles, bearing a charge of the same sign as that of the walls, into the body of the gas and into the neighbourhood of the electrode. In approaching the electrode they will induce on it a charge of such a sign that it appears to increase the leak due to the field; but since they move but slowly under the field, they will not increase the steady current appreciably. When the source of heat is removed, the convection currents become less violent and the particles sink again to the floor of the vessel ; in leaving the ncighbourhood of the electrode, they induce on it a charge of such a sign that it appears as a decrease of the steady leak. If the number of the particles is sufficiently great, the induced electrification may be great enough to cause the appearance of a temporary reversal of the current.

18. If this be indeed the true explanation, the heavy particles must have masses of an altogether different order to those of the ions. If $X$ is the electric intensity (seldom more than $\frac{1}{4}$ el. st. unit in these experiments), $e$ the charge, and $m$ the mass of a particle such that its weight is equal to the electrical force on it,

$$
\mathrm{X} e=m g, \quad \text { or } \quad e / m=\frac{981}{\frac{1}{4}}=3.9 \times 10^{3} .
$$

$e / m$ for an oxygen molecule is about $2 \times 13^{13}$ in the same units : lence the particles must contain about $5 \times 10^{9}$ molecules, the number which is contained in a sphere of air of $6 \times 10^{-4} \mathrm{cms}$. radius. If the effects did not increase with the ionization in the vessel, these particles might be safely viewed as fragments of the metal walls; but, as it is, it seems probable that they must consist, at least in part, of aggregations of the molecules of the gas.

19. Three attempts have been made to test the truth of this hypothesis.

(1) The air in the big vessel was stirred up by working a fan in the vessel by means of a shaft which passed through a stuffing-box in the side. If the heat-changes are due to convection currents, they should be reproduced by the action of the fan. It was thought that small changes of the nature expected were observed. In a series of 50 observations, the mean value of $t^{\prime}$ (just after starting the fan) was 5 per cent. less, and the mean value of $t^{\prime \prime}$ (just after stopping the fan) was 4 per cent. greater than the mean value of $t$. It was only to be expected that the effects should be small; for the currents produced by the fan differ markedly from 
those produced by heating in the feature which is most important for the present experiments, namely, the velocity of the current near the walls. On the other hand, it should be pointed out that the difficulty of getting the air in so lirge a vessel free from dust reduces the conclusiveness of the experiment.

20. (2) In the ressel shown in fig. 1 was placed a similarly shaped vessel of iron wire gauze with a mesh of $15 \mathrm{~cm}$. The gauze was supported on slate blocks, and was about $1.5 \mathrm{cms}$. distant from the walls of the vessel at each point; it could be maintained at any desired potential by means of a wire passing through the lid of the vessel. The gauze acted as an almost perfect electrostatic shield but permitted the passage of ions; when the walls and the gauze were at the same potential of \pm 500 volts, the leak was 30 per cent. greater than when the vessel was earthed and the gauze at \pm 500 volts.

When the vessel was heated with the walls and the gauze at the same potential, the changes observed were exactly similar to those when the gauze was absent except that the value of $Q$ was reduced by about 25 per cent. But when the gauze was at \pm 500 volts and the vessel earthed or at a potential differing still more from that of the gauze, the sign of the changes was reversed : the leak on initial heating was less, and that during initial cooling greater than the normal. The value of $Q$ is only about one-third of that observed in the absence of the gauze, and $Q^{\prime}$ is notably less than $Q$.

21. This difference is in eutire accordance with the explanation offered. Since the superficial area of the walls of the tin vessel is much greater than that of the wire in the walls of the gauze vessel, most of the particles will come from the tormer and their charge will be determined by the charge on the former. Since the P.D. between the walls and the electrode is less than that between the gauze and the electrode (it is the latter which determines the leak), the approach of the charged particles to the electrode will produce changes equivalent to a decrease of the leak. It will be seen that there must be some value of the potential of the walls for which the sign of the effects changes and their magnitude becomes zero. The value could only be estimated roughly; but it appears that the effects change sign when the P.D. between the electrode and the walls is about 100 volts less numerically than that between the electrode and the gauze.

22. (3) It was hoped that some light might be thrown on the subject by investigating the leak through the vessel immediately after the field was put on. A great many observations were taken, but the results were so inconsistent 
and unreliable that no conclusions of value could be drawn from them.

23. The matter cannot be regarded as definitely settled; but after trying almost every experiment, reasonable or unreasonable, that I can devise, I think that little more light can be thrown upon it by direct investigation.

My best thanks are due to Prof. Thomson for the innumerable helpful suggestions with which he has favoured me.

\section{Summary.}

24. The saturation current through the gas in a vessel which was alternately heated and cooled has been investigated. It was found that the current increased during the initial heating and decreased during the initial cooling.

The circumstances which determine the magnitude of these changes have been investigated.

It is suggested that the changes may be due to the induction of electrification on the electrode by very massive charged particles carried about by convection currents.

Cavendish Laboratory, Feb. 1906.

LII. Some Applications of the Theory of Electrons to the Theory of Phosphorescence. By JoskPH DE KowaLski, Dr. phil., Professor of Eaperimental Physics, University of Fribourg, Switzerland .

I. TTHE experiments performed by varisus scientists such 1 as Wiedemann, Leonard, Nichols, and Merritt, and others, demonstrate with increasing certitude that the difference between phosphorescence and. fluorescence is only apparent, and rather of a quintitative than of a qualitative nature.

In the presentation of the theory which follows, I have tried to correlate these phenomena and to find an explanation which accounts for them both on the same basis.

I intend to present only the general ideas of the theory and to indicate its utility in explaining the fundamental fucts in this domain. I will leave aside all details as I propose to study them apart.

I admit with Prof. J. J. Thomson that a system of corpuscules,

* Communicated by the Author.

This note is based on the ideas set forth by $\mathrm{P}_{\mathrm{r}}$ of. J. J. Thomson in 'Nature,' March 22, 1906. A summary thereof has been presented in a note to the Comptes Rendus de l'Académie des Sciences, Paris. 